\section{Was bestimmt Remission und niedrige Krankheits- aktivität bei SLE?}

Ugarte-Gil MF et al. Predictors of Remission and Low Disease Activity State in Systemic Lupus

Erythematosus: Data from a Multiethnic, Multinational Latin American Cohort. J Rheumatol 2019; 46: 1299-1308

Studienärzte der GLADEL-Studie suchten nach Prädiktoren für Remission und niedrigen Krankheitsaktivitätsstatus (LDAS) bei Patienten mit systemischem Lupus erythematodes (SLE), die nicht optimal kontrolliert wurden.

Für ihre Auswertung bestimmten die Ärzte aus 34 Zentren in 9 lateinamerikanischen Staaten zunächst 3 Krankheitszustände: eine Remission war definitionsgemäß erreicht, wenn der SLEDAI (Systemic Lupus Erythematosus Disease Activity Index) den Wert 0 erreichte und die Dosis Prednison $\leq 5 \mathrm{mg} /$ Tag betrug Immunsuppressiva als Erhaltungsdosis verabreicht wurden. Eine LDAS lag vor bei: SLEDAI $\leq 4$, Prednisondosis $\leq 7,5 \mathrm{mg} /$ Tag und/oder Immunsuppressiva als Erhaltungsdosis. Ein nicht optimal kontrollierter Zustand bedeutete: SLEDAI $>4$ und/oder Prednisondosis > 7,5 mg/Tag und/ oder Immunsuppressiva als Induktionsdosis. Antimalariamittel waren in allen Gruppen erlaubt. Die 3 Krankheitszustände wurden evaluiert zwischen 2 SLEDAI-Bestimmungen oder der letzten SLEDAI-Bestimmung und dem Ende der Nachbeobachtungsperiode. Eingeschlossen wurden nur diejenigen SLE-Patienten, die zum Zeitpunkt der ersten Bestimmung der Krank- heitsaktivität nicht optimal kontrolliert wurden, und bei denen mindestens eine weitere SLEDAI-Bestimmung erfolgte. Ergebnisse waren Remission und LDAS.

Von insgesamt 1480 überprüften Patientinnen und Patienten waren 902 beim Eintritt in die Studie nicht optimal kontrolliert. Diese Studiengruppe von 902 Patientinnen und Patienten war median 26 (20-36) Jahre alt und nahezu komplett weiblich ( $n=809$, $89,7 \%$ ). Die Nachbeobachtungszeit betrug median 56,3 Monate. Von diesen Patientinnen und Patienten erreichten 196 eine Remission (21,7\%), davon 97 über mindestens 3 Jahre und 47 eine anhaltende Remission. Eine LDAS erreichten 314 Patientinnen und Patienten (34,8\%).

Mithilfe der multivariablen Analyse wurden die Variablen identifiziert, die als Prädiktoren einer höheren Remissionswahrscheinlichkeit in Frage kamen. Dazu zählten: das Fehlen von mukokutanen Manifestationen (HR 1,571, 95 \% KI 1,064-2,320), das Fehlen einer Nierenbeteiligung (HR 1,487, $95 \%$ KI 1,067-2,073) und das Fehlen einer hämatologischen Beteiligung (HR 1,354 95\% KI 1,005-1,825). Weitere Prädiktoren waren der Einsatz von Immunsuppressiva vor der ersten Visite bei Studieneintritt (HR $1,468,95 \%$ KI 1,025-2,105) sowie ein niedrigerer SLEDAI-Wert bei Studienbeginn (HR 1,028, $95 \%$ KI 1,006-1,051 pro Abnahme einer Einheit).

Für eine niedrige Krankheitsaktivität(LDAS) waren die folgenden Parameter prädiktiv: älteres Alter bei Studieneintritt mit einer HR von 1,050 alle 5 Jahre (95\% KI 1,004$1,098)$. Das Fehlen von mukokutanen Manifestationen (HR 1,401, 95 \% KI 1,016$1,930)$ und eine Nierenbeteiligung (HR $1,344,95 \%$ KI 1,049-1,721) sowie ein niedrigerer SLEDAI-Score bei Studienbeginn (HR 1,025, 95 \% KI 1,009-1,042). 
FAZIT

Fehlende mukokutane, renale und hämatologische Manifestationen sowie der Einsatz von Immunsuppressiva und eine geringere Krankheitsaktivität zu Beginn des Krankheitsverlaufs waren prädiktiv für eine Remission bei Patienten mit SLE, so die GLADEL-Studienärzte. Für eine niedrige Krankheitsaktivität war ein höheres Alter ein Prädiktor.

Richard Kessing, Zeiskam 